\title{
Carnets
}

Revue électronique d'études françaises de l'APEF

Deuxième série - 19 | 2020

Petite fabrique d'interprètes

\section{Rivarol relu et corrigé}

De l'universalité à la diversité de la Langue Française

\section{Cristina Robalo Cordeiro}

\section{(2) OpenEdition}

Journals

Édition électronique

URL : http://journals.openedition.org/carnets/11392

DOI : $10.4000 /$ carnets. 11392

ISSN : 1646-7698

Éditeur

APEF

Référence électronique

Cristina Robalo Cordeiro, «Rivarol relu et corrigé », Carnets [En ligne], Deuxième série - 19 | 2020, mis en ligne le 31 mai 2020, consulté le 23 décembre 2020. URL : http://journals.openedition.org/carnets/ 11392 ; DOI : https://doi.org/10.4000/carnets. 11392

Ce document a été généré automatiquement le 23 décembre 2020.

\section{(c) (7) \&}

Carnets est mis à disposition selon les termes de la licence Creative Commons - Atribution - Pas d'utilisation commerciale 4.0 International. 


\title{
Rivarol relu et corrigé
}

\author{
De l'universalité à la diversité de la Langue Française
}

\section{Cristina Robalo Cordeiro}

1 Le "génie » de la langue française ou, pour prendre un mot moins connoté, "l'esprit" de la langue française, voilà un sujet qui m'a semblé convenir à l'occasion de cet hommage rendu à Maria Hermínia Laurel. Notre Collègue et notre Amie n'a-t-elle pas, comme moi et souvent avec moi, consacré le meilleur de sa vie professionnelle à la « défense et illustration de la langue française » - cette langue qu'elle possède à fond et des nombreuses littératures et cultures qui en sont le fruit?

2 Je songe à toutes les joies que nous ont données, depuis nos années d'études, vécues ensemble à la Faculté des Lettres de l'Université de Coimbra - il y a bientôt un demisiècle! -, cette langue, cette culture et cette littérature françaises régnant alors en souveraines absolues avant que le tiers-états des francophonies ne vienne revendiquer la place qu'elles occupent aujourd'hui dans les études et dans nos cœurs.

3 Que nous l'avons défendue, cette cause du français! Contre vents et marées, d'abord dans des amphithéâtres remplis d'étudiants zélés, qui se destinaient, presque tous, à être professeurs de français, et où on ne faisait que "prêcher des convertis", et bientôt, hélas, dans des salles désertées où tout ce qui nous était cher, tout ce qui nous semblait très précieux, s'est vu peu à peu dédaigné par une jeunesse désemparée à la recherche de filières moins compromises. Le désarroi qui a assombri la seconde moitié de nos carrières, assez semblable à l'angoisse du "petit reste » d'un culte abandonné, ce découragement profond, qui n'a cependant jamais entamé notre foi, il semble que nous en voyons le terme: de nombreux signes de renouveau sont perceptibles autour de nous, à commencer dans nos classes qui se repeuplent. Mais ne voyons pas dans ce regain de faveur un retour à un état de choses antérieur. Longtemps tributaire du prestige qu'elle avait acquis au Siècle des Lumières, la langue française s'est en effet à la fois démocratisée et modernisée. Jadis apanage d'une élite, aristocratique puis bourgeoise, elle a dû, en partie sous la pression politique et, sans doute, économique de la nouvelle « lingua franca », s'ouvrir aux peuples du monde, dont la plupart était enfin entré dans l'ère des indépendances. 
4 Je vais donc m'interroger sur ce qu'on appelle "l'esprit" de la langue française. Je ne le ferai pas en grammairienne ou en linguiste mais en m'attachant à définir les valeurs qu'elle a contribué et qu'elle contribuera encore, si nous le voulons tous, à faire respecter dans le monde. C'était hier l'universalité, c'est aujourd'hui la diversité. Mais et l'objection est souvent faite - n'y a-t-il pas dans ce passage de l'Un à l'Autre, plus qu'un retournement rhétorique : une véritable contradiction? J'essayerai d'en discuter après avoir passé un premier moment en compagnie du Comte de Rivarol, apologète de l'universalité à la toute fin de l'Ancien Régime, et un deuxième moment, trop rapide, avec la multitude bigarrée de tous ceux qui ont, en ce début $d u x x^{e}$ siècle, fait du français l'expression de leur "différence".

Deux mots d'abord sur ce curieux personnage qu'un texte d'une trentaine de pages, rédigé en 1783, paru en 1784, a rendu célèbre en quelques jours dans toutes les cours, dans tous les salons, clubs et cafés littéraires du vieux continent.

6 Le 6 juin 1782, l'Académie de Berlin avait mis au concours la, ou plutôt les questions suivantes: "Qu'est-ce qui a fait la langue française la langue universelle de l'Europe ? Par où mérite-t-elle cette prérogative ? Peut-on présumer qu'elle la conserve ?" Parmi la vingtaine de candidats se trouve un jeune homme de trente ans à peine, italien d'origine (son grand-père s'appelait Rivaroli). Six ans avant, quittant le Midi, il est monté à Paris et en arrivant dans la Capitale, riche de sa seule ambition, il s'est bientôt anobli, lui qui est né à l'Auberge des Trois Pigeons dans une famille de quinze enfants. Le nouveau Comte se fait apprécier de Voltaire qui, reconnaissant la vivacité de son intelligence, aussitôt le pousse dans le beau monde. Pour prouver son talent, il entreprend, sur le conseil de son protecteur, de traduire L'Enfer de Dante. C'est alors qu'il apprend qu'un prix sera donné à qui traitera le mieux le sujet proposé par Berlin. Il se met à la tâche, lit hâtivement tout ce qui pourra l'aider à répondre, compose, calligraphie et expédie son manuscrit, qui arrive à destination dans les derniers jours de 1783. De son côté, Schwab, un professeur allemand de l'Université de Stuttgart a envoyé un opuscule dix fois plus gros et plus savant. S'ils se partagent finalement le prix, malgré la disproportion des mérites, c'est que Henri, frère du Roi Frédéric II de Prusse, séduit par le brio du jeune français, a fait pression sur les membres du jury.

7 Du jour au lendemain, le pseudo Comte devient un personnage. Tout cela fait rêver. Avec plus de temps je pourrais m'amuser à comparer l'Académie littéraire de Berlin ou celle de Dijon (qui, trente ans avant, avait couronné le Discours de Jean-Jacques Rousseau sur les Arts et les Lettres) à l'académie de cinéma d'Hollywood.

Un tel rapprochement nous rappellerait surtout combien les réalités changent sous les mots qui restent: disons seulement qu'au $\mathrm{XVIII}^{\mathrm{e}}$ siècle on peut atteindre la gloire (procurée aujourd'hui par un "oscar") en écrivant une dissertation en français qui sera jugée par une académie allemande où l'on s'exprime en français.

9 Et Rivarol obtient, avec une médaille en or et cinquante ducats, le privilège d'être portraituré par le peintre Wyrsch qui nous a laissé de lui l'image d'un gentilhomme aux traits fins et à la moue satisfaite.

Nous sommes en 1784. Dans cinq ans ce sera la prise de la Bastille, dans huit ans la Terreur, dans onze ans le règne de Napoléon, dans vingt et un ans Waterloo et la fin des illusions impériales. Rivarol, ardent royaliste, va demeurer jusqu'au bout fidèle à Louis XvI, dont il sera le conseiller. Le roi s'était écrié, après avoir lu le Discours ${ }^{1}$ : "Ah ! comme cet auteur fait bien valoir la langue et la nation". 
11 Il faudrait bien sûr apporter de longues explications pour restituer l'atmosphère intellectuelle et culturelle où s'est inséré ce moment d'apothéose dans la vie de Rivarol et dans celle de la langue française, couronnée avec lui langue universelle de l'Europe. Ayant longtemps enseigné la Culture Française, je regrette de n'avoir jamais pensé à mettre l'étude du Discours de Rivarol au programme. Son étude attentive permettrait en effet d'avoir une vision panoramique et une sorte de bilan de la civilisation française et de son rayonnement au moment où va s'achever le Siècle des Lumières.

Je me contenterai, pour ma part, de dégager l'essentiel de son argumentation. Le discours est organisé autour des trois questions posées: la première partie, la plus longue, explique la genèse de la langue française et les raisons pour lesquelles elle a supplanté les langues des pays voisins. Ni l'allemand, ni l'italien, ni l'espagnol n'ont su s'imposer (j'observe qu'il n'est pas question du portugais!) malgré leurs qualités respectives, en remplacement du latin comme "lingua franca" des peuples chrétiens.

13 Quant à l'anglais, c'est la langue d'une Puissance jalouse, maîtresse des mers mais non pas des esprits et qui s'intéresse plus à ses "affaires", à son "commerce" qu'au bonheur des peuples. L'acrimonie de Rivarol à l'endroit de l'Angleterre (il venait de se séparer de sa femme: une Anglaise précisément...) le conduit à multiplier les jugements sommaires sur une nation où la France de l'époque voyait l'ennemi héréditaire.

14 Par opposition, l'Allemagne (encore divisée en une myriade de principautés) est l'Alliée naturelle. C'est du reste l'Allemagne, et plus précisément, la Prusse qui a permis à la langue française d'atteindre la position qu'elle occupe à la fin de l'Ancien Régime car Rivarol remarque que les nations du sud, Italie et Espagne, répugnent à la parler.

Quand nous lisons et disons Allemagne, Italie, Espagne prenons garde qu'il s'agit essentiellement des cours ou des milieux aristocratiques et savants, non des peuples de ces pays. Le suffrage universel, la démocratisation de l'enseignement, la massification de la culture changeront les données au cours du $\mathrm{XIX}^{\mathrm{e}}$ et plus encore du $\mathrm{XX}^{\mathrm{e}}$ siècle : ce qui a favorisé le français, tel que Rivarol à son époque en analyse la fortune, c'est l'idée de luxe auquel sa littérature était associée. Voici un passage révélateur :

Aux productions de l'esprit se joignaient encore celles de l'industrie : des pompons et des modes accompagnaient nos meilleurs livres chez l'étranger, parce qu'on voulait être partout raisonnable et frivole comme en France. Il arriva donc que nos voisins recevant sans cesse des meubles, des étoffes et des modes qui se renouvelaient sans cesse manquèrent de termes pour les exprimer: ils furent comme accablés sous l'exubérance de l'industrie française ; si bien qu'il prit comme une impatience générale à l'Europe, et que, pour n'être plus séparé de nous, on étudia notre langue de tous côtés. (D, § LII)

16 Autrement dit, c'est faute de pouvoir désigner dans leur propre langue les colifichets et brimborions de parure venus de Paris que les Européens se seraient mis à apprendre partout la langue du Roi-Soleil comme aujourd'hui c'est l'informatique avec sa nomenclature souvent intraduisible qui nous contraint à parler la langue de Bill Gates.

17 Mais si Rivarol n'est pas insensible à l'importance de la "frivolité" dans le succès du français, c'est au-delà de ce facteur contingent qu'il va découvrir la justification de son triomphe.

18 Influencé à la fois par Montesquieu et Buffon, il accorde au climat et aux époques de la nature un rôle fondamental. Le français est né et s'est développé dans une zone tempérée du globe et sa croissance a été favorisée par l'âge du Globe terrestre : si le climat change, le français est menacé dans son équilibre. Curieusement, certains 
aperçus du Discours annoncent ainsi nos préoccupations écologiques. Une langue, une culture sont à la merci des bouleversements du milieu naturel : en extrapolant un peu, on pourrait dire que le français est victime de la disparition de la couche d'ozone... Mais au $\mathrm{XVIII}^{\mathrm{e}}$ siècle, la planète ne connaissait pas encore les terribles effets de la pollution atmosphérique et le français fleurissait dans un air lumineux et pur entre les glaces du nord et les canicules australes.

Cependant il faut, avec la deuxième partie du Discours, examiner la structure logique de la langue française pour faire comprendre son "génie". Si le français mérite son statut privilégié de langue universelle, c'est qu'il est, tout simplement, la langue de la raison! Les idées claires et distinctes de Descartes sont comme chez elles dans la langue du Discours de la Méthode ou dans celle de la Monadologie, écrite en français comme d'autres traités de Leibnitz. Ce rationalisme naturel à la langue est pour ainsi dire passé en proverbes : c'est Rivarol lui-même qui propose cet adage (en lettres capitales dans le texte) : “CE QUI N’EST PAS CLAIR N’EST PAS FRANÇAIS”. Déjà Boileau avait prononcé dans son Art poétique la loi fameuse, devenue pierre de touche scolaire de l'esprit français: "Ce qui se conçoit bien s'énonce clairement". Les Romantiques et, plus encore, les Symbolistes se donneront beaucoup de mal pour rester français !

A la différence des langues à flexions, comme le latin ou l'allemand, le français consacre "l'ordre direct" (sujet-verbe-complément) qui est le mouvement le plus rationnel et le plus opposé au désordre des sensations et des émotions. Je cite à nouveau :

Le français, par un privilège unique, est seul resté fidèle à l'ordre direct, comme s'il était tout raison; et on a beau, par les mouvements les plus variés et toutes les ressources du style, déguiser cet ordre, il faut toujours qu'il existe; et c'est en vain que les passions nous bouleversent et nous sollicitent de suivre l'ordre des sensations: la syntaxe française est incorruptible. C'est de là que résulte cette admirable clarté, base éternelle de notre langue. (D, § LXVI)

21 Peu favorable à la poésie versifiée, Rivarol voit dans les images excessives, les métaphores filées des causes de corruption. C'est ainsi qu'il répond à la troisième question posée par le jury : très sévère pour les monstrueuses innovations de la Pléiade, préférant Racine à Molière, il condamne tout ce qui écarte l'écrivain de la saine sobriété et donc de la ligne droite du raisonnement.

Je ne m'attarderai pas sur cet intellectualisme néo-classique. Aristote avait lui aussi confondu les structures universelles de la logique avec celles propres à la langue grecque. Rivarol, partant de prémisses identiques, considère donc le français comme destiné par la Nature à servir de moyen d'expression aux sciences. Mais ce sont les grands écrivains qui ont préparé l'instrument.

23 En résumé, l'universalité de la langue française est celle même de l'esprit humain et quand il cherche la vérité dans les sciences, c'est en français qu'il la trouve et l'expose. Les diplomates, de leur côté, le savent bien : pour supprimer toute équivoque de la rédaction d'un traité, c'est, dit Rivarol, au français qu'ils doivent recourir.

On peut sourire de la prétention, parfois délirante, du Discours. Non seulement ce document ne peut se lire qu'à la lumière de l'histoire des idées et des mentalités, mais il présente le grand inconvénient de faire du français une essence fixe comme les espèces animales chez Buffon. Le concept d'évolution est encore inconnu, ou presque. On ne parle pas encore des dinosaures et de l'extinction des espèces. On sait toutefois que les langues meurent, avec les civilisations qui les ont produites. Et si le français devenait à 
son tour une langue morte? Rivarol envisage l'hypothèse mais son fixisme y voit une sorte de bienheureuse essentialisation ou de canonisation :

[...] Il suffit de dire que [les langues] après s'être élevées d'époque en époque jusqu'à la perfection, c'est en vain qu'elles en descendent: elles y sont fixées par les bons livres, et c'est en devenant langues mortes qu'elles se font réellement immortelles. (D. §LXXXVII)

C'est bien en effet la littérature qui assure le salut éternel des langues, surtout lorsque cette littérature s'est faite, comme la française, l'institutrice du genre humain. Mais voici la surprise. L'ethnocentrisme de Rivarol, au détour d'une page, bascule. L'auteur admet, non sans ironie, que le génie littéraire français pourrait se tarir. Alors il faudrait se tourner vers d'autres climats :

Les grands écrivains ont tout fait. Si notre France cessait d'en produire, la langue de Racine et de Voltaire deviendrait une langue morte; et si les Esquimaux nous offraient tout à coup douze écrivains du premier ordre, il faudrait bien que les regards de l'Europe se tournassent vers cette littérature des Esquimaux. (ibidem)

Il ne se doutait pas que l'avenir des langues européennes allait se jouer loin des frontières de l'Europe et que le français trouverait un jour dans le Maghreb, en Afrique Noire, dans l'Océan Indien, dans les Antilles des fontaines de jouvence dont il sortirait non pas abâtardi mais ragaillardi.

Il est vrai que, avec le désastreux traité de Paris, signé vingt ans avant le Discours, la langue française perdait l'Amérique et, par conséquent, la course à la souveraineté mondiale. Voltaire qui a tant fait pour la gloire de la France des Lumières n'a pas compris que l'avenir était, en partie, du côté de ces "quelques arpents de neige" auxquels il réduisait le Canada. Mais on ne refait pas l'histoire. Qui peut dire qu'une Amérique parlant le français et majoritairement catholique aurait connu le même développement?

Il serait utile d'examiner l'effondrement de l'idéal de Raison sous les coups de marteau du philosophe de la transmutation des valeurs, mais il me presse d'arriver à l'idée de diversité (très prisée par le même Nietzsche), valeur première de la francophonie. Il me suffirait pour l'illustrer de vous faire écouter l'enregistrement de tous les accents avec lesquels on parle le français hors de France (comme langue maternelle ou, en tout cas, apprise dans l'enfance): la variété des accents belge, suisse, québécois mais aussi tunisien ou algérien, marocain ou mauritanien, sénégalais ou congolais, malgache, mauricien, guadeloupéen, haïtien, etc., pour ne rien dire de toutes les voix qui s'élèvent de pays, comme la Roumanie, où le français a formé les élites intellectuelles depuis des siècles. Je signale qu'on parle le français depuis beaucoup plus longtemps à Beyrouth qu'en Bretagne. Sans m'engager dans la question difficile de la créolisation, je devrais évoquer cependant tous les enrichissements apportés au français "canonique" (celui sur lequel est censée veiller l'Académie française) par la bigarrure des mots empruntés aux langues locales. De la langue si forte de son grand roman Le Soleil des Indépendances le sénégalais Ahmadou Kourouma disait, en 1970, lors d'une interview : «Je l'ai pensé en malinké et écrit en français en prenant une liberté que j'estime naturelle avec la langue classique. [...] J’ai donc traduit le malinké en français, en cassant le français pour trouver et restituer le rythme africain ».

29 Les langues d'Afrique comme le lingala, le kikongo, le wolof, le peul, par exemple, ont déjà laissé leur empreinte sur le français des écrivains d'Afrique. Quant au français du Maghreb, il a déjà été richement illustré et vigoureusement défendu par des poètes et des romanciers de la stature du tunisien Albert Memmi, du marocain Driss Chraïbi, de 
l'algérien, déjà classique, Kateb Yacine. Tahar Ben Jelloun, qui s'est vu attribuer le prix Goncourt, a condensé sa conception et sa pratique d'auteur francophone dans une formule souvent reprise depuis: "J'écris pour dire la différence". L'entrée d'Assia Djebar d'abord à l'Académie Royale de Belgique, puis à l'Académie Française, en 2005, revêt l'éclat d'une double victoire : celle de la femme maghrébine et celle d'une grande écrivaine traduite dans le monde entier.

Cette différence et ces différences, on en retrouve l'affirmation sous la plume d'écrivains dispersés aux quatre coins de l'univers francophone : je ne suis pas la plus compétente ici pour entrer, si j'en avais le temps, dans le détail si séduisant des productions en français apparues dans l'Amérique du Nord : le Cajun de la Louisiane, le franco-ontarien, les parlers de l'Acadie et du Québec sont désormais aussi des langues littéraires. Tout comme les Antilles ont leur René Depestre, leur Aimé Césaire ou leur Edouard Glissant, maintenant mis au rang des classiques, entre des dizaines d'autres, morts ou vivants, jeunes ou vieux, qu'il faut mentionner.

31 Loin de moi pourtant l'idée de dresser un inventaire des noms et des œuvres qui font de la "francophonie" (je rappelle que le mot, dans son usage actuel, est dû à Léopold Senghor) un éventail versicolore. Du reste, la francophonie ne se dit-elle pas plus volontiers au pluriel? L'exercice ne serait pas fastidieux puisqu'il nous ferait voyager. Mais il resterait à en dégager la signification : que veut dire en effet cette diversité des langues et littératures que réunit la francophonie? Ne pourrait-on pas également - et avec autant de raisons - parler de la diversité de l'univers lusophone qui, en termes purement numériques, supplante assez largement la francophonie? En quoi la différence francophone est-elle différente des autres différences?

Je répondrai en un mot: Rivarol! C'est lui qui, applaudi par l'Europe savante de son temps, a montré que le génie de la langue française consistait dans l'universel intelligible que l'ordre syntaxique direct permet d'atteindre immédiatement. Langue abstraite, propice à l'expression des idées générales, le français se méfie des sensations - de ces sensations que Condillac a placées pourtant à l'origine de la pensée. L'idéalisme est l'idéologie, disons la métaphysique, inhérente au français : Victor Hugo puis Albert Camus ont su, chacun avec les éléments de son époque, honorer cette tradition des écrivains-penseurs de l'universel. Et c'est de l'interruption de cette lignée que se plaignent bien des amis de la France qui, dirait Victor Hugo, "devinant hélas l'avenir des colombes, pleure(nt) sur des berceaux et souri(ent) à des tombes". Mais pourquoi pleurer sur l'avenir quand, malgré nos nostalgies il nous sourit?

Un renversement de situation, ou plutôt une transmutation de valeurs s'est produite dans la seconde moitié $\mathrm{du} \mathrm{xx}^{\mathrm{e}}$ siècle. Une esthétique du divers s'est répandue et a fini par supplanter le rationalisme auquel on rattachait l'esprit français, l'esprit de la langue française. Au règne de l'universalité intelligible a succédé celui de la diversité sensible. Alors que le français du classicisme prônait l'identité et l'unité, le français contemporain nous est présenté, par les tenants de la francophonie plurielle, comme une langue ouverte à l'altérité. Entre-temps, certes, plus de trois siècles se sont écoulés et ce qui peut nous apparaître comme une antinomie en termes logiques cesse de nous choquer quand on la replace dans l'histoire, qui justifie tout. Et, depuis lors, que de dispositifs mis en place ('OIF, l'AUF, TV5 Monde, entre beaucoup d'autres) pour tenter d'harmoniser toutes ces voix qui veulent non seulement se faire entendre mais plus encore se faire écouter! 


\section{NOTES}

1. Rivarol. 1936. Discours sur l'universalité de la langue française, Paris: Classiques Larousse.

\section{RÉSUMÉS}

Cinq ans avant la Révolution française, le jeune Rivarol recevait le prix de l'Académie de Berlin pour son «Discours sur l'universalité de la langue française ». A l'apogée de son influence dans l'Europe des Lumières, la langue de Descartes et de Voltaire s'imposait comme la langue même de la Raison, accompagnée dans sa diffusion par les produits de luxe exportés de Paris. Relire aujourd'hui le Discours conduit à y apporter une correction majeure: l'avènement des francophonies et la crise de la rationalité européenne ont amené une telle transmutation de valeurs que la diversité concrète et vivante a plus de prix à nos yeux que l'universalité nécessairement abstraite et sans saveurs.

Five years before the French Revolution, young Rivarol received the Berlin Academy Prize for his "Discours sur l'universalité de la langue française". At the peak of its influence in the Europe of the Enlightenment, the idiom spoken by Descartes and Voltaire asserted itself as the very language of the Reason, supported by the luxury goods bought from Paris. Reading the Discours today leads us to make a major correction to its main assumption: the arising French-speaking countries and cultures and the crisis of the European Reason have brought forth such an axiological transformation that the concrete and living diversity has got in our eyes a much higher value than the abstract and tasteless universality of old. 
INDEX

Mots-clés : Rivarol (Antoine de), langue française, universalité, diversité, francophonie

Keywords : Rivarol (Antoine de), french language, universality, diversity, french-speaking world

\section{AUTEUR}

\section{CRISTINA ROBALO CORDEIRO}

Université de Coimbra

crobalo[at]uc.pt 